\title{
Boundary Stabilization of a Semilinear Wave Equation with Variable Coefficients under the Time-Varying and Nonlinear Feedback
}

\author{
Bei Gong ${ }^{1}$ and Xiaopeng Zhao ${ }^{2}$ \\ ${ }^{1}$ College of Computer Science, Beijing University of Technology, Beijing 100124, China \\ ${ }^{2}$ School of Science, Jiangnan University, Wuxi, Jiangsu 214122, China
}

Correspondence should be addressed to Bei Gong; tekkman_blade@163.com

Received 22 January 2014; Accepted 4 March 2014; Published 10 April 2014

Academic Editor: Dragoș-Pătru Covei

Copyright (C) 2014 B. Gong and X. Zhao. This is an open access article distributed under the Creative Commons Attribution License, which permits unrestricted use, distribution, and reproduction in any medium, provided the original work is properly cited.

We study the boundary stabilization of a semilinear wave equation with variable coefficients under the time-varying and nonlinear feedback. By the Riemannian geometry methods, we obtain the stability results of the system under suitable assumptions of the bound of the time-varying term and the nonlinearity of the nonlinear term.

\section{Introduction}

Many results concerning the boundary stabilization of classical wave equations are available in literatures. See [1-6] for linear cases and [7-14] for nonlinear ones. The stability of a nondissipative system described by partial differential equations (PDEs) has attracted much attention. Reference [15] developed the exponential stability for an abstract nondissipative linear system, and in [16], the Riesz basis property was developed for a beam equation with nondissipativity.

In [17], the following semilinear wave equation was considered:

$$
\begin{gathered}
u_{t t}-\Delta_{g} u+h(\nabla u)+f(u)=0 \quad(x, t) \in \Omega \times(0,+\infty), \\
\left.u(x, t)\right|_{\Gamma_{2}}=0 \quad t \in(0,+\infty), \\
\frac{\partial u(x, t)}{\partial \mu}+l\left(u_{t}\right)=0 \quad(x, t) \in \Gamma_{1} \times(0,+\infty), \\
u(x, 0)=u_{0}(x), \quad u_{t}(x, 0)=u_{1}(x) \quad x \in \Omega
\end{gathered}
$$

and the well-posedness and uniform decay of the energy of the system (1) was also established with linearly bounded $l(u)$ in [17].
Based on [17], we study the system (1) with time-varying and nonlinear feedback:

$$
\frac{\partial u(x, t)}{\partial \mu}+\phi(t) l(u)=0 \quad(x, t) \in \Gamma_{1} \times(0,+\infty) .
$$

The decay rate of the energy (when $t$ goes to infinity) of the wave equation with time-varying feedback was established under the assumption $\phi$ is decreasing [18-20] or $\phi$ has an upper bound [21].

In this paper, we consider the decay rate of the energy under suitable assumptions of the bound of the time-varying term $\phi(t)$ and the nonlinearity of the nonlinear term $l(u)$.

\section{Some Notation}

Let $\Omega$ be a bounded domain in $\mathbb{R}^{n}(n \geq 2)$ with smooth boundary $\Gamma$. It is assumed that $\Gamma$ consists of two parts $\Gamma_{1}$ and $\Gamma_{2}\left(\Gamma=\Gamma_{1} \cup \Gamma_{2}\right)$ with $\Gamma_{2} \neq \emptyset, \bar{\Gamma}_{1} \cap \bar{\Gamma}_{2}=\emptyset$.

Let $A(x)=\left(a_{i j}(x)\right)$ be symmetric, positively definite matrices for each $x \in \mathbb{R}^{n}$, and $a_{i j}(x)$ are smooth functions on $\mathbb{R}^{n}$. As in [22], we define

$$
g=A^{-1}(x) \quad \text { for } x \in \mathbb{R}^{n}
$$


as a Riemannian metric on $\mathbb{R}^{n}$ and consider the couple $\left(\mathbb{R}^{n}, g\right)$ as a Riemannian manifold with an inner product:

$$
\langle X, Y\rangle_{g}=\left\langle A^{-1}(x) X, Y\right\rangle, \quad|X|_{g}^{2}=\langle X, X\rangle_{g} \quad X, Y \in \mathbb{R}_{x}^{n} \text {. }
$$

Denote by $D, \nabla_{g}, \operatorname{div}_{g}$, and $\Delta_{g}$ the Levi-Civita connection, the gradient operator, the divergence operator, and the Beltrami-Laplace operator in terms of the Riemannian metric $g$, respectively. It can be easily shown that, under the Euclidean coordinate,

$$
\begin{gathered}
\nabla_{g} f=\sum_{i=1}^{n}\left(\sum_{j=1}^{n} a_{i j}(x) \frac{\partial}{\partial x_{j}} f\right) \frac{\partial}{\partial x_{i}}=A(x) \nabla f \\
\left|\nabla_{g} u\right|_{g}^{2}=\sum_{i, j=1}^{n} a_{i j}(x) \frac{\partial f}{\partial x_{i}} \frac{\partial f}{\partial x_{j}} \quad x \in \mathbb{R}^{n}, \\
\Delta_{g} f=\frac{1}{\sqrt{G}} \sum_{i=1}^{n} \frac{\partial}{\partial x_{i}}\left(\sqrt{G} \sum_{j=1}^{n} a_{i j}(x) \frac{\partial}{\partial x_{j}} f\right), \quad x \in \mathbb{R}^{n},
\end{gathered}
$$

where $\nabla f$ is the gradient of $f$ in the standard metric and $G=$ $\operatorname{det}(g)$.

Let $H$ be a vector field on $\left(\mathbb{R}_{x}^{n}, g\right)$. Then for each $x \in \mathbb{R}^{n}$, the covariant differential $D H$ of $H$ determines a bilinear form on $\mathbb{R}_{x}^{n}$ :

$$
D H(X, Y)=\left\langle D_{Y} H, X\right\rangle_{g} \quad \forall X, Y \in \mathbb{R}_{x}^{n},
$$

where $D_{Y} H$ stands for the covariant derivative of the vector field $H$ with respect to $Y$.

\section{The Main Results}

We consider the semilinear wave equation with variable coefficients under the time-varying and nonlinear boundary feedback:

$$
\begin{gathered}
u_{t t}-\Delta_{g} u+f(u)=0 \quad(x, t) \in \Omega \times(0,+\infty), \\
\left.u(x, t)\right|_{\Gamma_{2}}=0 \quad t \in(0,+\infty), \\
\frac{\partial u(x, t)}{\partial \mu}+\phi(t) l\left(u_{t}\right)=0 \quad(x, t) \in \Gamma_{1} \times(0,+\infty), \\
u(x, 0)=u_{0}(x), \quad u_{t}(x, 0)=u_{1}(x) \quad x \in \Omega,
\end{gathered}
$$

where $l, f$ are continuous nonlinear functions and $\mu(x)$ is the outside unit normal vector of the Riemannian manifold $(\Omega, g)$ for each $x \in \Gamma$. Different from [18-21], in this paper, we consider a general $\phi$; that is, $\phi \in C^{1}([0,+\infty))$ satisfies

$$
\frac{1}{\Phi(t)} \leq \phi \leq \Phi(t) \quad \forall t \geq 0,
$$

where $\Phi(t) \in C([0,+\infty))$ is a positive and nondecreasing function satisfying

$$
\lim _{t \rightarrow+\infty} \frac{\Phi(t)}{t}=0
$$

Let $\Phi^{\prime}(t) \in C([0,+\infty))$ be a positive and nondecreasing function with 0 as the limit. Then $t \Phi^{\prime}(t)$ satisfies (9). There are many examples of $\Phi^{\prime}(t)$ such as $(1+t)^{\alpha}(\alpha<0)$ and $e^{\beta t}(\beta<$ $0)$.

The main assumptions are listed as follows.

Assumption A. $f \in C^{1}(\mathbb{R}), f(0)=0$ derives from a potential F:

$$
F(s)=\int_{0}^{s} f(\tau) d \tau \geq 0 \quad \forall s \in \mathbb{R},
$$

and satisfies

$$
\left|f^{\prime}(s)\right| \leq b_{1}|s|^{\rho}+b_{2} \quad \forall s \in \mathbb{R},
$$

where $b_{1}, b_{2}$ are positive constants, and the parameter $\rho$ satisfies

$$
1 \leq \rho \leq \begin{cases}2, & n=2 \\ \frac{n}{n-2}, & n \geq 3\end{cases}
$$

Being different from [17], we assume the nonlinear term $l(u)$ has no growth restriction near zero as in $[23,24]$.

Assumption B. $l \in C^{1}(\mathbb{R})$ is a nondecreasing function satisfying

$$
l(0)=0, \quad c_{1}|s|^{2} \leq s l(s) \leq c_{2}|s|^{2} \quad \forall|s| \geq 1 .
$$

Assumption $C$. There exists a vector field $H$ on $\bar{\Omega}$ such that

$$
D H(X, X)=c(x)|X|_{g}^{2} \quad \text { for } X \in \mathbb{R}_{x}^{n} x \in \bar{\Omega},
$$

where $b=\min _{\bar{\Omega}} c(x)$ and $B=\max _{\bar{\Omega}} c(x)$

$$
B<\min \left\{b+\frac{2 b}{n}, r b\right\},
$$

where $r>1$ is a constant. Moreover we assume that

$$
\langle H, \mu\rangle_{g} \leq 0 \quad x \in \Gamma_{2}, \quad\langle H, \mu\rangle_{g} \geq 0 \quad x \in \Gamma_{1} .
$$

Condition (14) as a checkable assumption is very useful to study the control and stabilization of the wave equation with variable coefficients and the quasilinear wave equation [22, $25]$. For the examples of the condition, see $[22,26]$.

Based on condition (14), Assumption C was given by [17] to study the stabilization of the wave equation with variable coefficients and nonlinear boundary condition. Being different from [17], the lower bound of $\langle H, \mu\rangle_{g}$ was relaxed on $\Gamma_{1}$ from a positive constant to zero.

To facilitate the writing, we denote the volume element of $(\Omega, g)$ by $d x$ and denote the volume element of $(\Gamma, g)$ by $d \Gamma$. Define the energy of the system (7) by

$$
E(t)=\int_{\Omega}\left(u_{t}^{2}+\left|\nabla_{g} u\right|_{g}^{2}+2 F(u)\right) d x
$$

As in $[23,24]$, we let $h \in C([0,+\infty))$ be a concave increasing function such that

$$
h(0)=0, \quad s^{2}+(g(s))^{2} \leq h(s g(s)) \quad \text { for }|s| \leq 1 .
$$


With (18), the stabilization of the wave equation with variable coefficients and time dependent delay was studied by [27].

The main result of this paper is as follows.

Theorem 1. Let Assumptions A-C hold true. Assume that

$$
2 r F(s) \leq s f(s) \quad \forall s \in \mathbb{R},
$$

where $r$ is defined in (15).

(a) If the function l in (7) satisfies

$$
c_{1}|s|^{2} \leq s l(s) \leq c_{2}|s|^{2} \quad \forall|s|<1,
$$

then there exist constants $C>0$ such that

$$
E(t) \leq \frac{C \Phi(t)}{t} E(0) \quad t>0 .
$$

(b) If the functions $\phi(t), l$ in (7) satisfy

$$
\phi(t) \leq \phi_{0} \quad \forall t \geq 0, \quad s l(s) \geq c_{1}|s|^{2} \quad \forall|s|<1,
$$

where $\phi_{0}$ is a positive constant, then there exist constants $C_{1}, C_{2}>0$ such that

$$
C_{1} h\left(\frac{C_{2} \Phi(T)}{T} E(0)\right)+\frac{C_{1} \Phi(T)}{T} E(0) \quad t>0 .
$$

(c) If the function $\Phi(t)$ in (8) is a constant function; that is,

$$
\Phi(t)=\Phi(0) \quad \forall t \geq 0,
$$

then there exist constants $C_{1}, C_{2}>0$ such that

$$
C_{1} h\left(\frac{C_{2} E(0)}{T}\right)+\frac{C_{1}}{T} E(0) \quad t>0 .
$$

\section{Well Posedness of the System}

Define

$$
H_{\Gamma_{2}}^{1}(\Omega)=\left\{u \in H^{1}|(\Omega) u|_{\Gamma_{2}}=0\right\} .
$$

By a similar proof as Lemma 7.1 in [17], we have the following result.

Theorem 2. Let Assumptions $A-B$ hold true. For any initial data $\left(u_{0}, u_{1}\right) \in H_{\Gamma_{2}}^{1}(\Omega) \times L^{2}(\Omega)$, system (7) admits a unique weak solution $u$ such that $u \in C\left([0,+\infty), H_{\Gamma_{2}}^{1}(\Omega)\right) \cap$ $C^{1}\left([0,+\infty), L^{2}(\Omega)\right)$. Define

To prove Theorem 1, we still need several lemmas further.

$$
E_{0}(t)=\int_{\Omega}\left(u_{t}^{2}+\left|\nabla_{g} u\right|_{g}^{2}\right) d x
$$

Then, we have

$$
E(t)=E_{0}(t)+2 \int_{\Omega} F(u) d x .
$$

The following lemma shows the energy of the system (7) is decreasing.
Lemma 3. Suppose that Assumptions A-B hold true. Let $u$ be the solution of the system (7). Then

$$
E(0)-E(T)=2 \int_{0}^{T} \int_{\Gamma_{1}} \phi(t) u_{t} l\left(u_{t}\right) d \Gamma d t .
$$

The assertion (29) implies that $E(t)$ is decreasing.

Proof. Differentiating (17), we obtain

$$
\begin{aligned}
E^{\prime}(t) & =\int_{\Omega}\left(2 u_{t} u_{t t}+2\left\langle\nabla_{g} u, \nabla_{g} u_{t}\right\rangle_{g}+2 f(u)\right) d x \\
& =\int_{\Gamma_{1}} 2 \phi(t) u_{t} l\left(u_{t}\right) d \Gamma .
\end{aligned}
$$

Then the inequality (29) follows directly from (30) integrating from 0 to $T$.

\section{Proofs of Theorem 1}

Lemma 4. Let $u(x, t)$ be the solution of the equation $u_{t t}+\Delta q u+$ $f(u)=0,(x, t) \in \Omega \times(0,+\infty)$ and that $\mathscr{H}$ is a vector field defined on $\bar{\Omega}$. Then for $T \geq 0$

$$
\begin{gathered}
\int_{0}^{T} \int_{\Gamma} \frac{\partial u}{\partial \mu} \mathscr{H}(u) d \Gamma d t+\frac{1}{2} \int_{0}^{T} \int_{\Gamma}\left(u_{t}^{2}-\left|\nabla_{g} u\right|_{g}^{2}-2 F(u)\right) \\
\times\langle\mathscr{H}, \mu\rangle_{g} d \Gamma d t \\
=\left.\left(u_{t}, \mathscr{H}(u)\right)\right|_{0} ^{T}+\int_{0}^{T} \int_{\Omega} D \mathscr{H}\left(\nabla_{g} u, \nabla_{g} u\right) d x d t \\
+\frac{1}{2} \int_{0}^{T} \int_{\Omega}\left(u_{t}^{2}-\left|\nabla_{g} u\right|_{g}^{2}-2 F(u)\right) \operatorname{div}_{g} \mathscr{H} d x d t .
\end{gathered}
$$

Moreover, assume that $P \in C^{1}(\bar{\Omega})$. Then

$$
\begin{aligned}
\int_{0}^{T} \int_{\Omega} & \left(u_{t}^{2}-\left|\nabla_{g} u\right|_{g}^{2}-u f(u)\right) P d x d t \\
= & \left.\left(u_{t}, u P\right)\right|_{0} ^{T}+\frac{1}{2} \int_{0}^{T} \int_{\Omega} \nabla_{g} P\left(u^{2}\right) d x d t \\
& -\int_{0}^{T} \int_{\Gamma} P u \frac{\partial u}{\partial \mu} d \Gamma d t .
\end{aligned}
$$

Proof. Note that

$$
\mathscr{H}(u) f(u)=\mathscr{H}(F(u))=\operatorname{div}_{g}(F(u) \mathscr{H})-F(u) \operatorname{div}_{g} \mathscr{H} .
$$

The equality (31) and the equality (32) follow from Proposition 2.1 in [22].

Lemma 5. Suppose that all assumptions in Theorem 1 hold true. Let $u$ solve the system (7). Then there exist positive constants $\bar{T}, C$ for which

$$
E(T) \leq \frac{C}{T} \int_{0}^{T} \int_{\Gamma_{1}}\left(u_{t}^{2}+\left(\frac{\partial u}{\partial \mu}\right)^{2}\right) d \Gamma d t,
$$

where $T \geq \bar{T}$. 
Proof. From (15), we choose a positive constant $\theta$ satisfying

$$
\theta<\frac{n b}{2}, \quad b+\theta-\frac{n B}{2}>0, \quad 2 r \theta>n B .
$$

Set

$$
\mathscr{H}=H, \quad P=\theta .
$$

We substitute the formula (32) into the formula (31), and we have

$$
\begin{aligned}
\Pi_{\Gamma}= & \left.\left(u_{t}, H(u)+P u\right)\right|_{0} ^{T} \\
& +\int_{0}^{T} \int_{\Omega}\left(D H\left(\nabla_{g} u, \nabla_{g} u\right)-b\left|\nabla_{g} u\right|_{g}^{2}\right) d x d t \\
& +\int_{0}^{T} \int_{\Omega}\left(\left(\frac{1}{2} \operatorname{div} H-\theta\right) u_{t}^{2}\right. \\
& \left.+\left(b+\theta-\frac{1}{2} \operatorname{div} H\right)\left|\nabla_{g} u\right|_{g}^{2}\right) d x d t \\
& +\int_{0}^{T} \int_{\Omega}[\theta(u f(u)-2 r F(u)) \\
& +(2 r \theta-\operatorname{div} H) F(u)] d x d t,
\end{aligned}
$$

where

$$
\begin{aligned}
\Pi_{\Gamma}=\int_{0}^{T} \int_{\Gamma} & \frac{\partial u}{\partial \mu}(H(u)+u P) d \Gamma d t \\
& +\frac{1}{2} \int_{0}^{T} \int_{\Gamma}\left(u_{t}^{2}-\left|\nabla_{g} u\right|_{g}^{2}-2 F(u)\right)\langle H, \mu\rangle_{g} d \Gamma d t .
\end{aligned}
$$

Decompose $\Pi_{\Gamma}$ as

$$
\Pi_{\Gamma}=\Pi_{\Gamma_{1}}+\Pi_{\Gamma_{2}}
$$

where $\Pi_{\Gamma_{1}}\left(\Pi_{\Gamma_{2}}\right)$ stands by the value of the terms on the right side of (38) integrating on $\Gamma_{1}\left(\Gamma_{2}\right)$.

Similar to $[5,22]$, we deal with $\Pi_{\Gamma_{2}}$ as follows.

Since $\left.u\right|_{\Gamma_{2}}=0$, we have $\left.\nabla_{\Gamma} u\right|_{\Gamma_{2}}=0$; that is,

$$
\nabla_{g} u=\frac{\partial u}{\partial \mu} \mu \quad \text { for } x \in \Gamma_{2} \text {. }
$$

Similarly, we obtain

$$
H(u)=\left\langle H, \nabla_{g} u\right\rangle_{g}=\frac{\partial u}{\partial \mu}\langle H, \mu\rangle_{g} \quad \text { for } x \in \Gamma_{2} .
$$

Using the equality (40) and (41) in the equality (38) on the portion $\Gamma_{2}$, with (16) we obtain

$$
\Pi_{\Gamma_{2}}=\frac{1}{2} \int_{0}^{T} \int_{\Gamma_{2}}\left(\frac{\partial u}{\partial \mu}\right)^{2}\langle H, \mu\rangle_{g} d \Gamma d t \leq 0 .
$$

Let $H_{1}$ be a vector field on $\bar{\Omega}$ such that

$$
\begin{aligned}
& H_{1}=\mu \quad x \in \Gamma_{1}, \\
& H_{1}=0 \quad x \in \Gamma_{2} .
\end{aligned}
$$

Set $\mathscr{H}=H_{1}$; it follows from (31) that

$$
\begin{aligned}
\int_{0}^{T} \int_{\Gamma_{1}}\left(\frac{\partial u}{\partial \mu}\right)^{2} d \Gamma d t+\frac{1}{2} \int_{0}^{T} \int_{\Gamma_{1}}\left(u_{t}^{2}-\left|\nabla_{g} u\right|_{g}^{2}\right) d \Gamma d t \\
=\left.\left(u_{t}, H_{1}(u)\right)\right|_{0} ^{T}+\int_{0}^{T} d t \int_{\Omega} D H_{1}\left(\nabla_{g} u, \nabla_{g} u\right) d x \\
+\frac{1}{2} \int_{0}^{T} d t \int_{\Omega}\left(u_{t}^{2}-\left|\nabla_{g} u\right|_{g}^{2}-2 F(u)\right) \operatorname{div}_{g} H_{1} d x .
\end{aligned}
$$

Then we obtain that

$$
\begin{aligned}
& \int_{0}^{T} \int_{\Gamma_{1}}\left|\nabla_{g} u\right|_{g}^{2} d \Gamma d t \\
& \leq C \int_{0}^{T} \int_{\Gamma_{1}}\left(u_{t}^{2}+\left(\frac{\partial u}{\partial \mu}\right)^{2}\right) d \Gamma d t+C\left(E_{0}(0)+E_{0}(T)\right) \\
& \quad+C \int_{0}^{T} \int_{\Omega}\left(u_{t}^{2}+\left|\nabla_{g} u\right|_{g}^{2}+2 F(u)\right) d x d t .
\end{aligned}
$$

With (16) and (45), we have

$$
\begin{aligned}
\Pi_{\Gamma_{1}}= & \int_{0}^{T} \int_{\Gamma_{1}} \frac{\partial u}{\partial \mu}(H(u)+u P) d \Gamma d t \\
& +\frac{1}{2} \int_{0}^{T} \int_{\Gamma_{1}}\left(u_{t}^{2}-\left|\nabla_{g} u\right|_{g}^{2}-F(u)\right)\langle H, \mu\rangle_{g} d \Gamma d t \\
\leq & C_{\varepsilon} \int_{0}^{T} \int_{\Gamma_{1}}\left(\frac{\partial u}{\partial \mu}\right)^{2} d \Gamma d t+\varepsilon \int_{0}^{T} \int_{\Gamma_{1}}\left(u^{2}+\left|\nabla_{g} u\right|_{g}^{2}\right) d \Gamma d t \\
& +C \int_{0}^{T} \int_{\Gamma_{1}} u_{t}^{2} d \Gamma d t \\
\leq & C \int_{0}^{T} \int_{\Gamma_{1}}\left(\frac{\partial u}{\partial \mu}\right)^{2} d \Gamma d t \\
& +\varepsilon\left(E_{0}(0)+E_{0}(T)+\int_{0}^{T} E(t) d t\right)+C \int_{0}^{T} \int_{\Gamma_{1}} u_{t}^{2} d \Gamma d t .
\end{aligned}
$$

Note that

$$
n b \leq \operatorname{div}_{g} H \leq n B \quad \forall x \in \bar{\Omega} .
$$

Substituting the formulas (42) and (46) into the formula (37), with (19) and (35), we obtain

$$
\begin{aligned}
\int_{0}^{T} E(t) d t \leq & C\left(E_{0}(0)+E_{0}(T)\right) \\
& +C \int_{0}^{T} \int_{\Gamma_{1}}\left(u_{t}^{2}+\left(\frac{\partial u}{\partial \mu}\right)^{2}\right) d \Gamma d t .
\end{aligned}
$$


Since

$$
\begin{aligned}
E_{0}(0) & =E_{0}(T)-\int_{0}^{T} \int_{\Gamma_{1}} u_{t} \frac{\partial u}{\partial \mu} d \Gamma d t \\
& \leq E_{0}(T)+\frac{1}{2} \int_{0}^{T} \int_{\Gamma_{1}}\left(u_{t}^{2}+\left(\frac{\partial u}{\partial \mu}\right)^{2}\right) d \Gamma d t
\end{aligned}
$$

from (48), we have

$$
\int_{0}^{T} E(t) d t \leq C E(T)+C \int_{0}^{T} \int_{\Gamma_{1}}\left(u_{t}^{2}+\left(\frac{\partial u}{\partial \mu}\right)^{2}\right) d \Gamma d t .
$$

Since $E(t)$ is decreasing, we deduce that

$$
\int_{0}^{T} E(t) d t \geq T E(T)
$$

Substituting the formulas (51) into the formula (50), for sufficiently large $T$, we have

$$
E(T) \leq \frac{C}{T} \int_{0}^{T} \int_{\Gamma_{1}}\left(u_{t}^{2}+\left(\frac{\partial u}{\partial \mu}\right)^{2}\right) d \Gamma d t .
$$

The inequality (34) holds.

Proof of Theorem 1. (a) From (8), (13), (20), (29), and (34), for $T \geq \bar{T}$ we deduce that

$$
\begin{aligned}
E(T) \leq & \frac{C}{T} \int_{0}^{T} \int_{\Gamma_{1}}\left(\phi^{2}(t)+1\right) u_{t}^{2} d \Gamma d t \\
\leq & \frac{C}{T}(\sup \{\phi(t) \mid 0 \leq t \leq T\} \\
& \left.\quad+\sup \left\{\frac{1}{\phi(t)} \mid 0 \leq t \leq T\right\}\right) \\
& \times \int_{0}^{T} \int_{\Gamma_{1}} \phi(t) u_{t}^{2} d \Gamma d t \leq \frac{C \Phi(T)}{T} E(0) .
\end{aligned}
$$

Note that $E(t)$ is decreasing, and the estimate (21) holds.

(b) From (8), (13), (22), (29), and (34), for $T \geq \bar{T}$ we deduce that

$$
\begin{array}{r}
E(T) \leq \frac{C}{T} \int_{0}^{T} \int_{\Gamma_{1}}\left(\phi^{2}(t) g^{2}\left(u_{t}\right)+u_{t}^{2}\right) d \Gamma d t \\
\leq \frac{C}{T}\left\{\int_{0}^{T} \int_{\Gamma_{1}} \phi(t) g^{2}\left(u_{t}\right) d \Gamma d t\right. \\
\left.+\Phi(T) \int_{0}^{T} \int_{\Gamma_{1}} \phi(t) u_{t}^{2} d \Gamma d t\right\}
\end{array}
$$

$$
\begin{aligned}
\leq & \frac{C}{T}\left\{\int_{0}^{T} \int_{\left\{x \in \Gamma_{1},\left|u_{t}\right| \leq 1\right\}} \phi(t) g^{2}\left(u_{t}\right) d \Gamma d t\right. \\
& \left.+\Phi(T) \int_{0}^{T} \int_{\Gamma_{1}} \phi(t) u_{t} g\left(u_{t}\right) d \Gamma d t\right\} \\
\leq & \frac{C}{T} \int_{0}^{T} \int_{\left\{x \in \Gamma_{1},\left|u_{t}\right| \leq 1\right\}} \phi(t) h\left(u_{t} g\left(u_{t}\right)\right) d \Gamma d t \\
& \quad \frac{C \Phi(T)}{T} E(0) \\
\leq & \frac{C}{T} \int_{0}^{T} \int_{\Gamma_{1}} \phi(t) h\left(u_{t} g\left(u_{t}\right)\right) d \Gamma d t+\frac{C \Phi(T)}{T} E(0) \\
\leq & \frac{C \int_{0}^{T} \phi(t) d t \cdot \operatorname{meas}\left(\Gamma_{1}\right)}{T} h \\
& \times\left(\frac{\int_{0}^{T} \int_{\Gamma_{1}} \phi(t) u_{t} g\left(u_{t}\right) d \Gamma d t}{\int_{0}^{T} \phi(t) d t \cdot \operatorname{meas}\left(\Gamma_{1}\right)}\right)+\frac{C \Phi(T)}{T} E(0) \\
\leq & C_{1} h\left(\frac{C_{2} \Phi(T)}{T} E(0)\right)+\frac{C_{1} \Phi(T)}{T} E(0) .
\end{aligned}
$$

Note that $E(t)$ is decreasing, and the estimate (23) holds.

(c) From (8), (13), (24), (29), and (34), for $T \geq \bar{T}$ we deduce that

$$
\begin{aligned}
E(T) \leq & \frac{C}{T} \int_{0}^{T} \int_{\Gamma_{1}}\left(\phi^{2}(t) g^{2}\left(u_{t}\right)+u_{t}^{2}\right) d \Gamma d t \\
\leq & \frac{C}{T} \int_{0}^{T} \int_{\Gamma_{1}} \phi(t)\left(g^{2}\left(u_{t}\right)+u_{t}^{2}\right) d \Gamma d t \\
\leq & \frac{C}{T} \int_{0}^{T} \int_{\left\{x \in \Gamma_{1},\left|u_{t}\right| \leq 1\right\}} \phi(t) h\left(u_{t} g\left(u_{t}\right)\right) d \Gamma d t \\
& +\frac{C}{T} \int_{0}^{T} \int_{\left\{x \in \Gamma_{1},\left|u_{t}\right|>1\right\}} \phi(t) u_{t}^{2} d \Gamma d t \\
\leq & \frac{C}{T} \int_{0}^{T} \int_{\Gamma_{1}} \phi(t) h\left(u_{t} g\left(u_{t}\right)\right) d \Gamma d t \\
\leq & \frac{C \int_{0}^{T} \phi(t) d t \cdot \operatorname{meas}\left(\Gamma_{1}\right)}{T} h \int_{0}^{T} \int_{\Gamma_{1}} \phi(t) u_{t} g\left(u_{t}\right) d \Gamma d t \\
& \times\left(\frac{\int_{0}^{T} \int_{\Gamma_{1}} \phi(t) u_{t} g\left(u_{t}\right) d \Gamma d t}{\int_{0}^{T} \phi(t) d t \cdot \operatorname{meas}\left(\Gamma_{1}\right)}\right)+\frac{C}{T} E(0) \\
\leq & C_{1} h\left(\frac{C_{2} E(0)}{T}\right)+\frac{C_{1}}{T} E(0) .
\end{aligned}
$$

Note that $E(t)$ is decreasing, and the estimate (25) holds. 


\section{Conflict of Interests}

The authors declare that there is no conflict of interests regarding the publication of this paper.

\section{Acknowledgments}

The authors would like to express their deep thanks to Zhen-Hu Ning for bringing conditions (8) and (16) to us as the requirements for the stability results in Theorem 1 . The research is supported by the Open Fund of Trust Computing Laboratory of Beijing University of Technology Start-up Fund of Beijing University of Technology under Grants (007000543113522).

\section{References}

[1] J. E. Lagnese, "Note on boundary stabilization of wave equations," SIAM Journal on Control and Optimization, vol. 26, no. 5, pp. 1250-1256, 1988.

[2] D. L. Russell, "Controllability and stabilizability theory for linear partial differential equations: recent progress and open questions," SIAM Review, vol. 20, pp. 639-739, 1978.

[3] R. Triggiani, "Wave equation on a bounded domain with boundary dissipation: an operator approach," Journal of Mathematical Analysis and Applications, vol. 137, no. 2, pp. 438-461, 1989.

[4] Y. You, "Energy decay and exact controllability for the Petrovsky equation in a bounded domain," Advances in Applied Mathematics, vol. 11, no. 3, pp. 372-388, 1990.

[5] Z.-H. Ning and Q.-X. Yan, "Stabilization of the wave equation with variable coefficients and a delay in dissipative boundary feedback," Journal of Mathematical Analysis and Applications, vol. 367, no. 1, pp. 167-173, 2010.

[6] Z. H. Ning, C. X. Shen, and X. P. Zhao, "Stabilization of the wave equation with variable coeffcients and a delay in dissipative internal feedback," Journal of Mathematical Analysis and Applications, vol. 405, no. 1, pp. 148-155, 2013.

[7] M. Aassila, M. M. Cavalcanti, and V. N. Domingos Cavalcanti, "Existence and uniform decay of the wave equation with nonlinear boundary damping and boundary memory source term," Calculus of Variations and Partial Differential Equations, vol. 15, no. 2, pp. 155-180, 2002.

[8] M. M. Cavalcanti, V. N. Domingos Cavalcanti, and P. Martinez, "Existence and decay rate estimates for the wave equation with nonlinear boundary damping and source term," Journal of Differential Equations, vol. 203, no. 1, pp. 119-158, 2004.

[9] F. Conrad and B. Rao, "Decay of solutions of the wave equation in a star-shaped domain with nonlinear boundary feedback," Asymptotic Analysis, vol. 7, no. 3, pp. 159-177, 1993.

[10] V. Komornik, "On the nonlinear boundary stabilization of the wave equation," Chinese Annals of Mathematics B, vol. 14, no. 2, pp. 153-164, 1993.

[11] V. Komornik, Exact Controllability and Stabilization: The Multiplier Method, John Wiley and Sons. Ltd, Chichester, UK, 1994.

[12] V. Komornik and E. Zuazua, "A direct method for the boundary stabilization of wave equation," Journal de Mathématiques Pures et Appliquées, vol. 69, pp. 33-54, 1990.

[13] I. Lasiecka and D. Tataru, "Uniform boundary stabilization of semilinear wave equation with nonlinear boundary condition," Differential Integral Equations, vol. 6, pp. 507-533, 1993.
[14] E. Zuazua, "Uniform stabilization of the wave equation by nonlinear boundary feedback," SIAM Journal on Control and Optimization, vol. 28, no. 2, pp. 466-477, 1990.

[15] K. S. Liu, Z. Y. Liu, and B. Rao, "Exponential stability of an abstract nondissipative linear system," SIAM Journal on Control and Optimization, vol. 40, no. 1, pp. 149-165, 2002.

[16] B. Z. Guo, J. M. Wang, and S. P. Yung, "On the C0-semigroup generation and exponential stability resulting from a shear force feedback on a rotating beam," Systems \& Control Letters, vol. 18, no. 6, pp. 1013-1038, 2005.

[17] B.-Z. Guo and Z.-C. Shao, "Exponential stability of a semilinear wave equation with variable coeffcients under the nonlinear boundary feedback," Nonlinear Analysis, vol. 71, pp. 5961-5978, 2009.

[18] M. Bellassoued, "Decay of solutions of the wave equation with arbitrary localized nonlinear damping," Journal of Differential Equations, vol. 211, no. 2, pp. 303-332, 2005.

[19] A. Benaissa and A. Guesmia, "Energy decay for wave equations of $\varphi$-Laplacian type with weakly nonlinear dissipation," Electronic Journal of Differential Equations, vol. 109, pp. 1-22, 2008.

[20] A. Benaissa, A. Benaissa, and S. A. Messaoudi, "Global existence and energy decay of solutions for the wave equation with a time varying delay term in the weakly nonlinear internal feedbacks," Journal of Mathematical Physics, vol. 53, no. 12, Article ID 123514, 2012.

[21] H. Li, C. S. Lin, S. P. Wang, and Y. M. Zhang, "Stabilization of the wave equation with boundary time-varying delay," Advances in Mathematical Physics, vol. 2014, Article ID 735341, 6 pages, 2014.

[22] P.-F. Yao, "On the observability inequalities for exact controllability of wave equations with variable coefficients," SIAM Journal on Control and Optimization, vol. 37, no. 5, pp. 15681599, 1999.

[23] I. Lasiecka and D. Tataru, "Uniform boundary stabilization of semilinear wave equation with nonlinear boundary dissipation," Differential Integral Equations, vol. 6, pp. 507-533, 1993.

[24] M. M. Cavalcanti, V. N. Domingos Cavalcanti, and I. Lasiecka, "Well-posedness and optimal decay rates for the wave equation with nonlinear boundary damping-source interaction," Journal of Differential Equations, vol. 236, no. 2, pp. 407-459, 2007.

[25] P. F. Yao, "Boundary controllability for the quasilinear wave equation," Applied Mathematics and Optimization, vol. 61, no. 2, pp. 191-233, 2010.

[26] P. F. Yao, Modeling and Control in Vibrational and Structural Dynamics. A Differential Geometric Approach, Applied Mathematics and Nonlinear Science Series, Chapman and Hall/CRC CRC Press, Boca Raton, Fla, USA, 2011.

[27] Z. H. Ning, C. X. Shen, X. P. Zhao, H. Li, C. S. Lin, and Y. M. Zhang, "Nonlinear Boundary Stabilization of the Wave Equations with Variable coeffcients and time dependent delay," Applied Mathematics and Computation, vol. 232, pp. 511-520, 2014. 


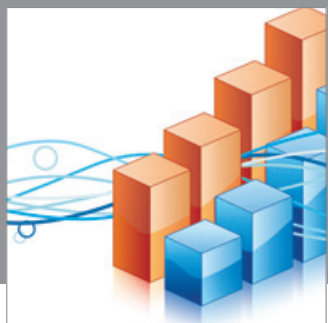

Advances in

Operations Research

mansans

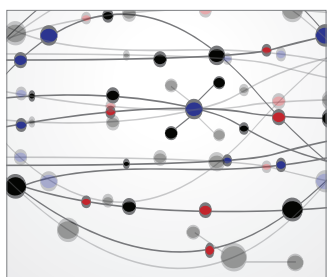

The Scientific World Journal
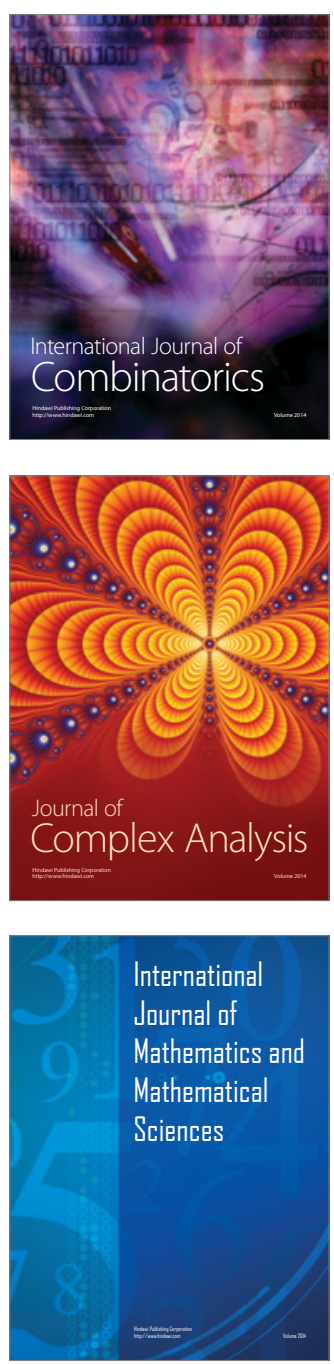
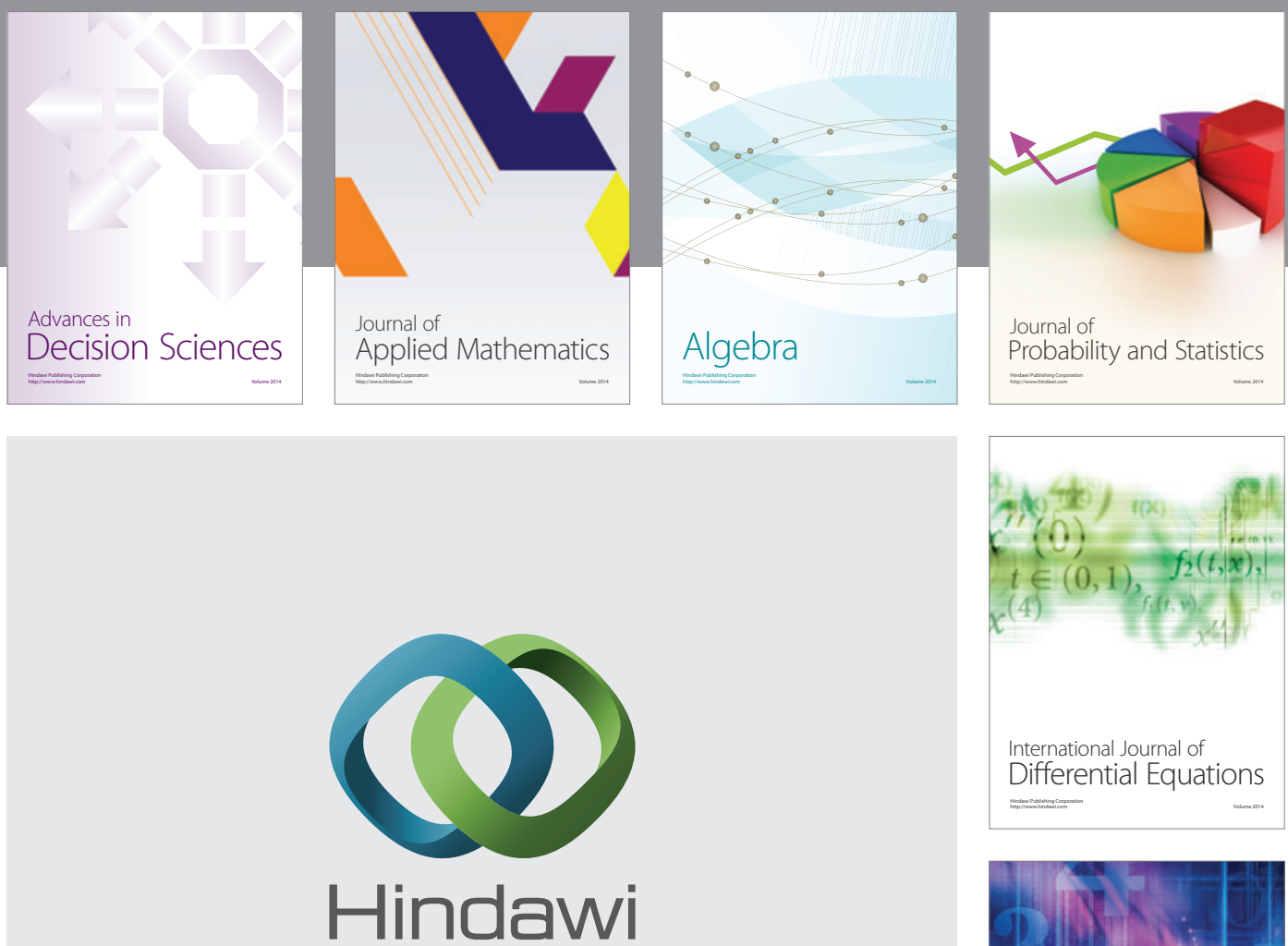

Submit your manuscripts at http://www.hindawi.com
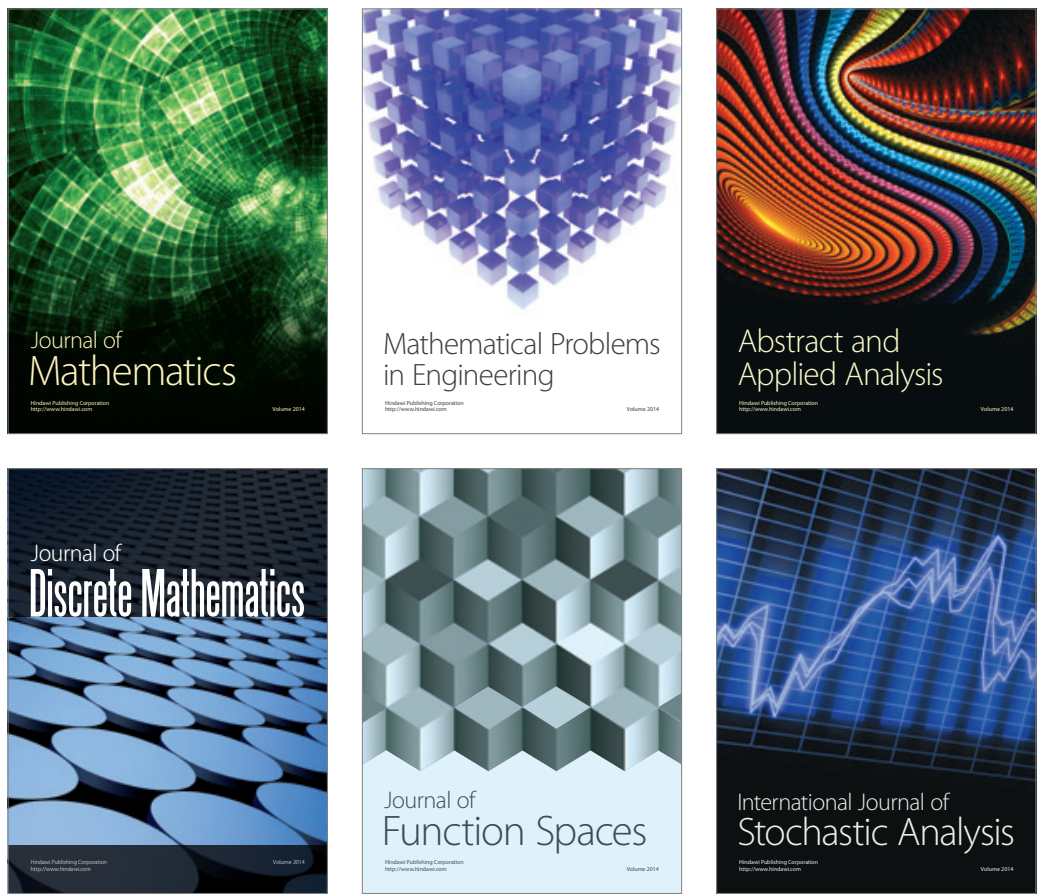

Journal of

Function Spaces

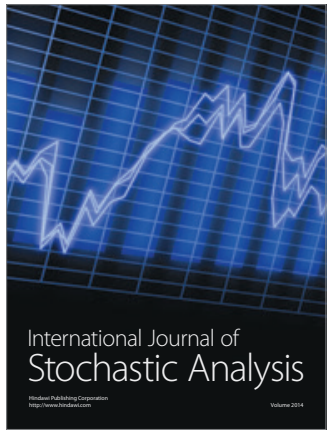

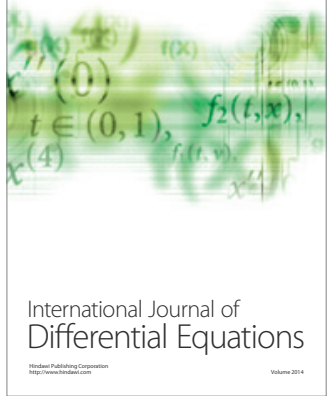
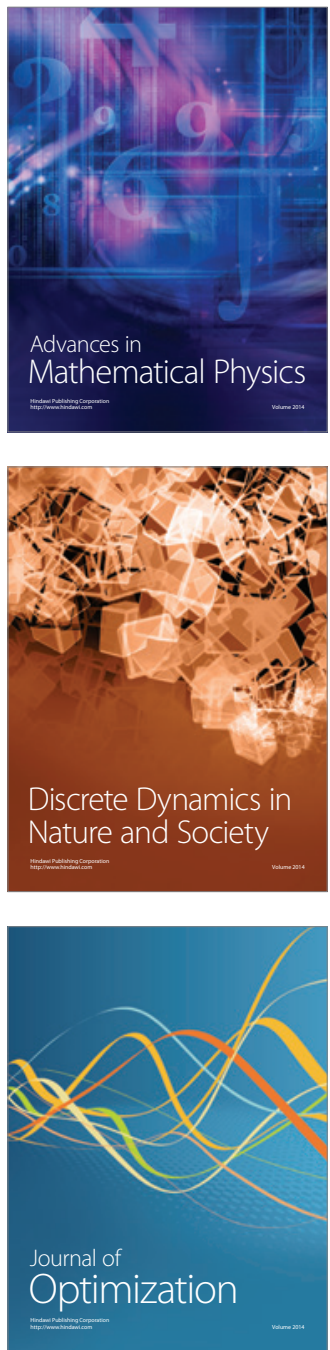